\section{Graphene and other materials highlighted at 2010 Materials Research Society Fall Meeting}

\author{
www.mrs.org/fall2010
}

T

2010 Materials Research Society (MRS) Fall Meeting was held in Boston on November 28-December 3. Meeting Chairs Ana Claudia Arias (Palo Alto Research Center), Robert F. Cook (National Institute of Standards and Technology), Clemens Heske (University of Nevada, Las Vegas), and Shu Yang (University of Pennsylvania) compiled a program of 50 technical symposia, broadly divided into the areas of materials for information processing; materials for infrastructure and mechanical applications; materials processing and device fabrication; materials for energy, biological and environmental applications; and exploration of novel materials and their properties.

In addition to reports on research results, which is the mainstay of MRS Meetings overall, the Fall Meeting served as host to many other functions of the Society, including public outreach, professional development, and networking across continents in the mission of building the materials field.
Dave Ginley, the 2010 MRS president, paid tribute to the memory of Rustum Roy (the Pennsylvania State University), one of the founders of MRS and president in 1977, who died last summer. Roy stood for interdisciplinarity, and was an innovative thinker, visionary, and an outstanding materials researcher.

Following are some of the research highlights reported at the Meeting. For further details, see the symposium summaries posted on the MRS website at www.mrs.org/bulletinfall2010. In addition, proceedings as well as additional meeting highlights are available at website www.mrs.org/fall2010.

\section{Graphene}

Graphene is among the carbon-based nanomaterials emerging as leading candidates for the fabrication of a new generation of high-performance nanoelectronic devices. This material, which exhibits extraordinary physical properties, received much attention at the Fall Meeting. One of the two 2010 physics

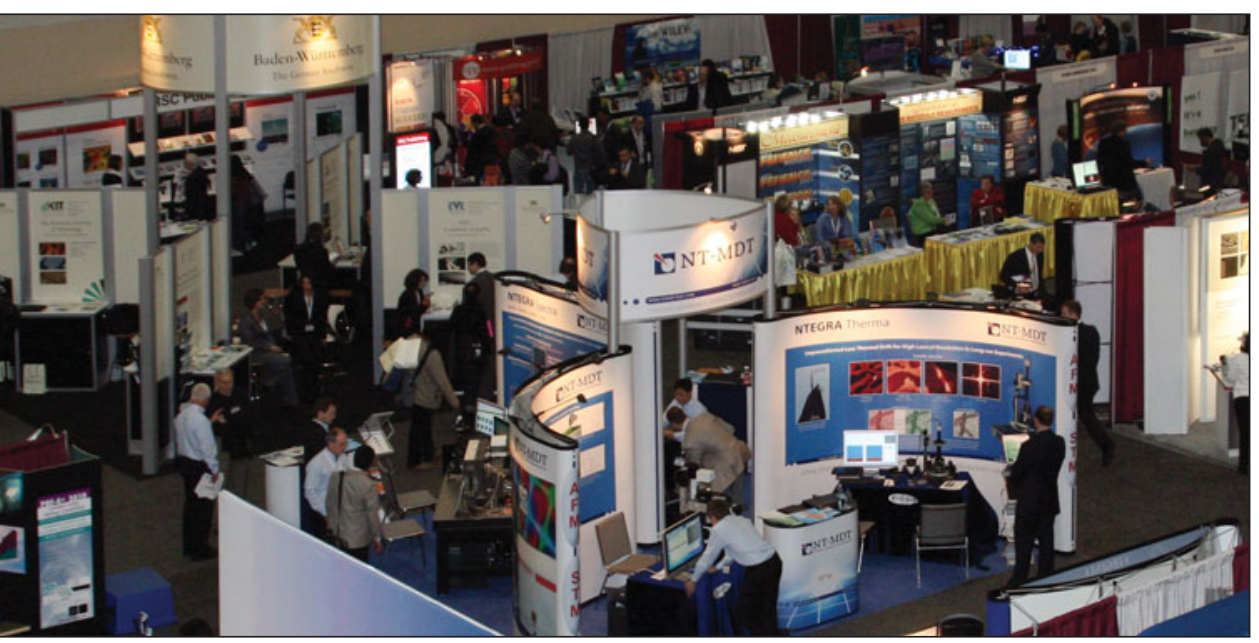

Nobel laureates, Konstantin S. Novoselov (University of Manchester, UK), as well as 2010 MRS Medalist Walter A. de Heer (Georgia Institute of Technology) presented their seminal work on this material. The material was further discussed in various symposia, including in an invited talk by Phaedon Avouris, (IBM T.J. Watson Research Center) who focused on potential applications of graphene in electronics.

Novoselov, who shares the 2010 Nobel Prize in physics with Andre Geim (University of Manchester, UK), reviewed graphene's properties: that the electrons move over large, submicron distances as if they were free of the environment just a few angstroms away; and that the electrons are governed by the Dirac equation, not the Schrödinger equation, mimicking relativistic particles with no rest mass. Novoselov reviewed applications of graphene, including transistors, quantum dots, variable capacitors, and photovoltaics. Reaction with hydrogen turns the semi-metallic graphene into graphane - an insulator, which is crystalline and retains the hexagonal lattice. The reaction is reversible, however, so that annealing restores the original metallic state. Graphene can therefore be considered to be a huge, molecular scaffold onto which other atoms can be attached, resulting in new, two-dimensional crystals with designed electronic properties. Novoselov said the challenge for graphene's most interesting applications lies in combining its various superlative properties.

De Heer described structured epitaxial graphene where the aim is to "grow it in the shape we want it in." He used a stepped $\mathrm{SiC}$ substrate, which is fairly easy to make lithographically, and showed that graphene grows preferentially on the steps. Similarly, a SiC substrate with a round mesa was fabricated. Again, graphene grew preferentially on the step, forming a graphene ring. An ar- 
ticle based on de Heer's Medalist presentation is forthcoming in MRS Bulletin.

Avouris addressed how graphene can be used in electronic and photonic devices. The zero bandgap of the material means that carriers cannot be completely confined, so graphene is not a good candidate for devices with digital switching. However, Avouris presented strategies for opening a bandgap in graphene; for example, for graphene bilayers, an electric field can make the potential of each layer different. Although the induced bandgap is still not large enough for digital switching, bilayer transistors using graphene operating at room temperature were demonstrated. Avouris also showed results for a graphene-based photodetector used for the reliable detection of 10-GBit/s optical data streams.

\section{Materials for information processing}

Symposia D, F, and G shared a session particularly dedicated to novel manufacturing issues for organic electronic devices. The direction of the talks covered aspects of the value chain comprising device architecture under manufacturing constraints, process development, materials supply (including ink formulation), device characterization, and examples for potential market applications.

P. Blom (TNO/Holst Center, Eindhoven, The Netherlands), in his presentation on roll-to-roll fabricated organic devices, cited the statistic that $>19 \%$ of all generated electricity is used for lighting. Organic light-emitting diodes (OLEDs), he said, have the potential to revolutionize the lighting market. OLEDs fabricated on flexible foils have several beneficial properties, including tunable color, large emitting surfaces, high efficiency/low operational cost, freedom of form, and are much more difficult to break compared to lighting technologies that use glass. However, the layer-quality requirements for OLEDs are more stringent than for organic photovoltaics. For example, visual defects will result from significant layer inhomogeneities. In addition, good encapsulation of the OLED is necessary because they are very sensitive to moisture and oxygen.
The Holst Center's roadmap to OLEDs made with roll-to-roll processing starts with an ITO-free cathode design. Finite element modeling was used to optimize the design of the silver grid, which is in a honeycomb pattern. The thin silver lines are susceptible to shunting, so they must be embedded, which posed a problem for depositing the active layer with inkjet printing; a homogeneous film is deposited on a heterogeneous substrate. An alternative approach used was slotdie coating. Subsequent steps in the fabrication process included lamination and thin-film encapsulation. Blom showed a movie in which an OLED was cut in half. Both halves still illuminated. One of the halves was then cut in half, and it recovered its illumination after a short time, although some parts of the cut edges had dark spots. One of the first applications for the flexible OLED was a rear-view mirror on a race car that finished fourth in the LeMans 2010, although, as Blom said, "it only needed to last 24 hours."

H. Sirringhaus (University of Cambridge, UK) processed metal-oxide fieldeffect transistors (FETs) from solution

\section{Materials Research Society Fall Meeting} Symposium Support

3D Micromac AG

3M Company

Advanced Diamond Technologies, Inc.

AGD Material Co. Ltd.

Agilent Technologies

Air Force Office of Scientific Research

Air Force Research Laboratory

AIXTRON AG

AlphaMicron

Angstrom Engineering

Apollo Diamond

Applied Diamond, Inc.

Argonne National Laboratory

Army Research Office

Aspen Aerogels, Inc

Asylum Research

Binghamton University-State University

of New York

Blake Industries

Cabot Corp.

CEA-LIST

CEA-Saclay

Center for Integrated Nanotechnologies,

Los Alamos and Sandia National

Laboratories

Companhia Brasileria de Metalurgia

e Mineracão (CBMM)

Deutsche Forschungsgemeinschaft, DFG

Element Six

FEl Company

General Electric Global Research

Georgia Tech Research Institute

Hysitron, Inc.

IBM T.J. Watson Research Center

INOSTEK, Inc.

Inst. for Materials Research, Hasselt University and IMOMEC Division IMEC

Institute of Physics v.v.i., Academy of Sciences of the Czech Republic

Insync, Inc.

JEOL USA, Inc

K-TEK Nanotechnology

Kleindiek Nanotechnik GmbH

The Kurt J. Lesker Co.

Lambda Technologies, Inc.

Lawrence Livermore National Laboratory

Mad City Labs., Inc.
Marlow Industries, Inc.

Millipore Corporation

MORESCO Co., Ltd.

Nanofactory Instruments

Nanomechanics, Inc.

National Institute for Materials Science

(NIMS), Japan

National Institute of Standards and Technology

National Science Foundation

NETSCH Instruments N.A. LLC

Ningbo Institute of Materials Technology

\& Engineering (NIMTE), China

NSF Division of Materials ResearchSolid-State Chemistry

Office of Naval Research

Palo Alto Research Center

Park Systems Corporation

PARU Co., Ltd.

PicoQuant/OEC

Plansee SE

Plextronics, Inc.

Princeton Instruments

Protochips, Inc.

Quantum Design, Inc.

Renewable and Sustainable Energy Inst.

(RASEI), a joint Institute between the Univ. of Colorado at Boulder and NREL

Rigaku Americas Corp.

Robert Bosch LLC Research

and Technology Center

Sandia National Labs, Center for Integrated

Nanotechnologies

Seki Technotron USA \& sp3 Diamond

Technologies

SOLVAY S.A.

SPECS Surface Nano Analysis GmbH

SPS Syntex, Inc.

Tanaka Kikinzoku Kogyo K.K.

Thermal Technology LLC

Thin Film Electronics ASA

Tohoku University

University of Maryland, Dept. of MS\&E

University of Maryland, Materials Research

Science \& Engineering Ctr.

Veeco Instruments

Xerox Corporation 

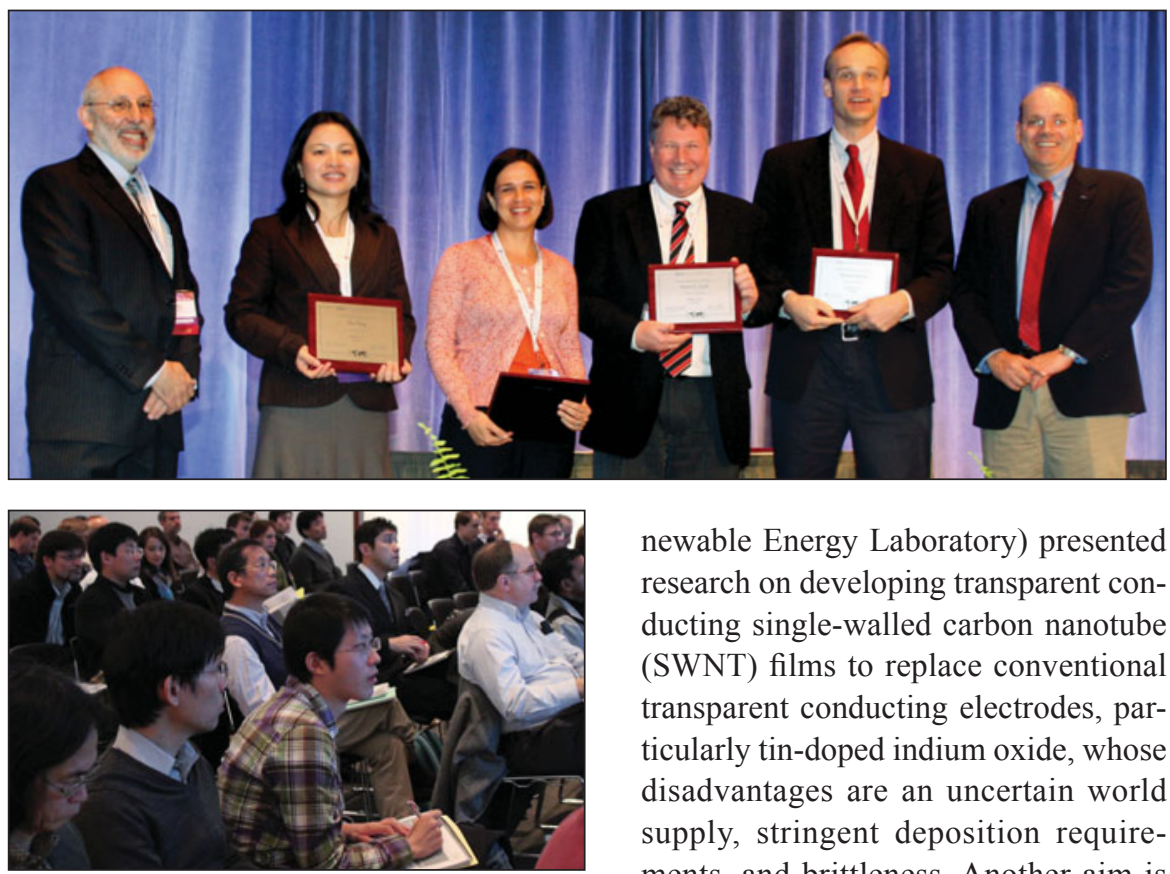

at moderate temperature conditions. He introduced a sol-gel hydrolysis process (wet annealing) of the inorganic films from ternary or quarternary metaloxides precursors and showed that it is possible to achieve reasonable FET output characteristics at annealing temperatures as low as $210^{\circ} \mathrm{C}$. Therefore, solution processing of inorganic films turns out to be an alternative to purely organic electronics approaches.

M. Poliks (State University of New York, Binghamton) outlined a roll-to-roll machinery concept comprising vacuum deposition, photolithography, and wet deposition. Through an integrated manufacturing concept, low-cost niche applications such as intravascular ultrasound transducers or optical waveguides on plastic substrates with channel widths of $30 \mu \mathrm{m}$ could be achieved. Furthermore, T. Yokota (University of Tokyo, Japan) described organic transistors with a $3 \mathrm{~V}$ operational voltage obtained using a subfemtoliter electrostatic inkjet, achieving micron structure widths.

There is considerable interest in chemically based materials systems that allow the fabrication of electronic devices such as efficient solar-energy harvesters, light emitters, transistors, or sensors. In symposium E on molecular and hybrid materials for electronics and photonics, J. Blackburn (National Re- newable Energy Laboratory) presented research on developing transparent conducting single-walled carbon nanotube (SWNT) films to replace conventional transparent conducting electrodes, particularly tin-doped indium oxide, whose disadvantages are an uncertain world supply, stringent deposition requirements, and brittleness. Another aim is replacing the electron-accepting fullerene phase in organic photovoltaic (OPV) solar cells with SWNTs. Blackburn introduced a versatile and scalable ultrasonic spray process for producing transparent SWNT electrodes with high conductivity. Adaptable to aqueous as well as organic solvents, this process can be used to apply SWNTs to a variety of substrates. In actual devices incorporating these new SWNT electrodes, performance is nearly equivalent to that of traditional devices with traditional electrodes made of typical transparent conducting oxides. Blackburn presented general results of time-resolved microwave spectroscopic studies demonstrating long-lived charge separation at the interfaces formed between SWNTs and the semiconducting polymer poly-3(hexylthiophene), which is important for the successful replacement of the fullerene phase in OPV solar cells with SWNTs.

With the growth of liquid-crystaldisplay (LCD) televisions and other display devices around the world, research is now turning to next-generation LC materials including their utilization in nontraditional applications, as presented in symposium L. For example, C. van Oosten presented the latest progress made by his company, Peer+ in the Netherlands, on an energy-efficient window that controls light based on sunlight condi- tions. Oosten's Smart Energy Glass is an LC-based technology that works through a fluorescent guest-host system where a fluorescent dye controls light transmission and a luminescent solar concentrator, whose principle was discovered in the late 1970s, collects and provides power. In the "off" state, a super-twisted liquid crystal is nematically aligned, while in the high-voltage state the helix is unwound. A "fingerprint" texture describes the intermediate state. Smart Energy Glass powers itself and has three states: bright, dark, and privacy. It is also easy to install and has low maintenance. Remaining challenges include transport loss that currently limits the window size to about a $1 \times 1.5 \mathrm{~m}^{2}$, which is still suitable for the majority of applications but, Oosten said, "Technology can be scaled up rapidly using existing manufacturing techniques from the LCD industry."

Symposium organizer T. White (Air Force Research Laboratory), who served as a substitute for invited speaker B. Taheri (AlphaMicron Inc., Ohio), discussed light-induced crystal order, which is a new paradigm for LC materials. LCs are typically categorized as thermotropic, lyotropic, and polymeric depending on how liquid crystallinity is induced. The new category-phototropic LCs - are similar to lyotropic and thermotropic LCs in that transitions from the isotropic phase to nematic, cholesteric, and smectic phases are observed; however, incident light induces liquid-crystalline order in phototropic liquid crystals. A novel class of photoinduced, dichroic dyes were synthesized, which, when added to certain conventional liquid crystals, induced the phototropic effect. White said that under appropriate illumination, a transition from an order parameter of zero to an order parameter observed in conventional liquid crystals was observed. White contrasted this result to conventional azo dyes that are typically added to LCs, wherein light illumination disrupts liquid-crystalline order. In addition, the new phototropic liquid crystalline system exhibits no spatial phase separation, that is, the system as a whole exhibits the phototropic effect.

Recent advances in nanofabrication 
have enabled rapid developments in the area of optical materials. Given the strong analogies with communication antennas in the microwave and radio-wave regime, a new class of resonant optical antennas has emerged. M.A. Noginov (Norfolk State University, Virginia), in symposium $\mathrm{M}$, expressed optimism that plasmonic metamaterials will revolutionize information technology by merging the advantages of modern compact electronics with those of fast photonics. $\mathrm{He}$ said that the coupling of photons, surface plasmons, and electrons is key for photonic nanocircuitry. Presenting a survey of his work in this area, Noginov discussed photo-excitation of macroscopic electric currents in silver films under conditions where surface plasmon polaritons were excited in the Kretschmann geometry as well as in configurations where surface plasmon polaritons were not excited. By propagating electric current in metallic films, Noginov showed that surface plasmon polaritons are controlled externally. The experimental setup included silver films 35-60 nm thick, deposited on high-index glass prisms and glass slides. Surface plasmon polaritons were excited when laser light entered the films from one of the sides of the prism, caused by a sudden decrease in the angular dependence of the reflectance. The direction of the electric signal induced by the laser illumination for on-resonance incidence angles was opposite to that induced by off-resonance incidence angles, although the magnitude of the electrical signal at resonant excitation far exceeded that of the off-resonant signal.

\section{Materials for infrastructure and mechanical applications}

Despite the use of nanoscale building blocks in next-generation technological devices, which are often subjected to extreme stresses during operation, a complete understanding of the governing deformation mechanisms at these length scales still remains elusive. Size-dependent strengthening in crystalline materials has been the subject of vigorous research, although the effect of finite and pre-existing materials defects on mechanical response has seen relatively little attention.

\section{Poster prizes awarded at 2010 MRS Fall Meeting}

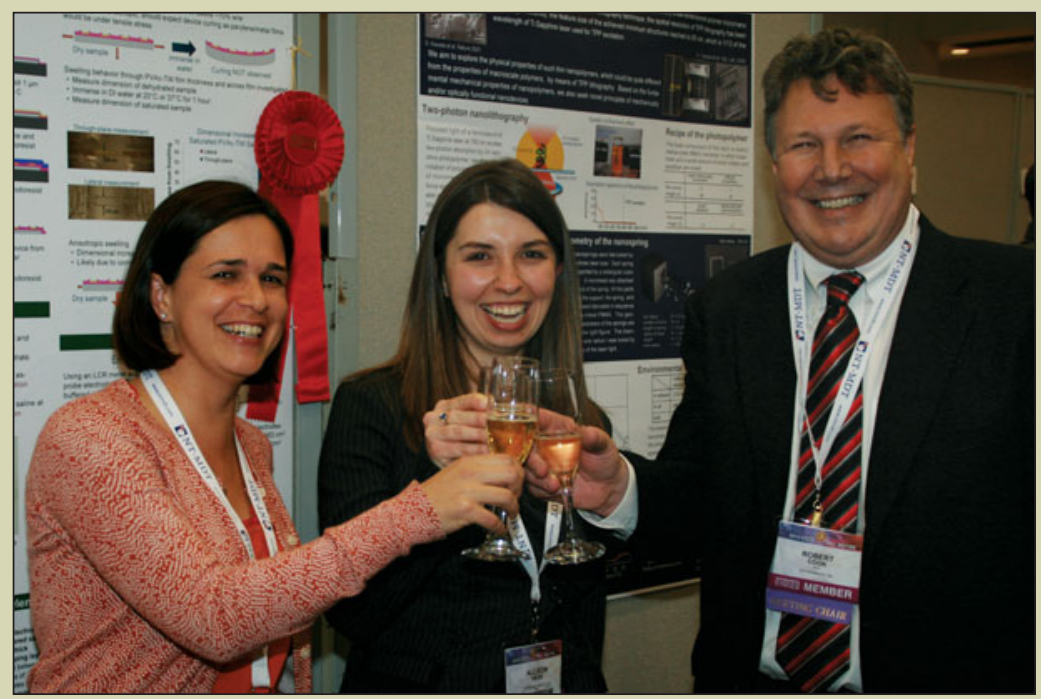

The 2010 Fall Meeting Chairs awarded prizes for the following best poster presentations: (C10.11) The role of the dielectric constant for the excitonic transition energy of singlewall carbon nanotubes, P.T. Araujo and A. Jorio of the Universidade Federal de Minas Gerais, Brazil;, A.R. Nugraha, K. Sato, and R. Saito of Tohoku University, Japan; and M.S. Dresselhaus of the Massachusetts Institute of Technology,USA; (E8.40) Singlet exciton fission in organic solar cells, P. Jadhav, A. Mohanty, J. Sussman, and M. Baldo of the Massachusetts Institute of Technology, USA; (E11.28) Flexible nonvolatile memory devices based on organic thin film transistors and self-assembled metallic nanoparticles, S.-J. Kim, Y.-S. Park, S.-H. Lyu, J.-M. Song, and J.-S. Lee of Kookmin University, South Korea; (H5.2) Hairloss in nanocrystals: Controlling nanocrystal superlattice symmetry and shape-anisotropic interactions through variable ligand surface coverage, J.J. Choi, C.R. Bealing, K. Bian, R.G. Hennig, and T. Hanrath of Cornell University, USA and

D.M. Smilgies of Cornell High Energy Synchrotron Source, USA; (L9.10) Effect of interdigitation on phase changes in side-chain liquid crystalline polymers bearing cholesterol, S.-K. Ahn and R.M. Kasi of the University of Connecticut, USA; (M10.9) Enhancing the optical properties and chemical stability of plasmonic nanoholes using atomic layer deposition of dielectric overlayers, H. Im, N.C. Lindquist, A. Lesuffleur, and S.-H. Oh of the University of Minnesota, USA; (N5.38) Structure analysis of a long period stacking ordered phase in Mg-Al-Gd alloys, $\mathrm{H}$. Yokobayashi, K. Kishida, and H. Inui of Kyoto University, Japan and M. Yamasaki and Y. Kawamura of Kumamoto University, Japan; (P3.10) Mechanisms of stress-driven motion of asymmetrical tilt grain boundaries, Z. Trautt and Y. Mishin of George Mason University, USA; (P10.44) Plasticity controlled nanoscale wear in SiC, M. Mishra and I. Szlufarska of the University of Wisconsin-Madison,USA; (S4.7) Fabrication and characterization of MEMS-based structures from a bio-inspired, chemo-responsive polymer nanocomposite, A. Hess, S.J. Rowan, and C.A. Zorman of Case Western Reserve University; J.R. Capadona and D.J. Tyler of the Louis Stokes Veterans Affairs Medical Center and Case Western Reserve University; and C. Weder of Case Western Reserve University and the University of Fribourg, Switzerland; (U9.6) Molecular dynamics modeling of the structure and radiation response of amorphous solids, R.E. Baumer and M.J. Demkowicz of the Massachusetts Institute of Technology, USA and T. Oppelstrup and V. Bulatov of Lawrence Livermore National Laboratory, USA; (W6.12) Non-volatile resistive switching effect in limited nanospace of a single $\mathrm{NiO}$ heterostructured nanowire, K. Oka and K. Nagashima of Osaka University, Japan; T. Yanagida of Osaka University and the Japan Science and Technology Agency, Japan; J.-S. Kim and B.-H. Park of Konkuk University, South Korea; and T. Kawai of Osaka University, Japan and Konkuk University, South Korea; (Y3.13) FIB-assisted molecular beam epitaxy and optical properties of metalsemiconductor nanocomposites, M. Kang, J.-H. Wu, and R.S. Goldman of the University of Michigan, USA; (HH3.15) New insights into solvated block copolymer networks as nanostructured dielectric elastomers, A.S. Krishnan, T.K. Ghosh, and R.J. Spontak of North Carolina State University, USA and S. Seifert and B. Lee of Argonne National Laboratory, USA; (JJ5.5) Development of a particle nano-imprinting technique by coreshell particles, H. Watanabe and K. Fujimoto of Keio University, Japan; (MM6.21) Structural and electrical characterization of oxide spinels as candidates for $p$-type transparent conductors, N.H. Perry, A.R. Nagaraja, and T.O. Mason of Northwestern University, USA and J.S. Bettinger and M.F. Toney of the SLAC National Accelerator Laboratory, USA; (0013.18) Freeze-cast chitosan-hydroxyapatite bone substitute materials and scaffolds support osteoblast attachment, proliferation and differentiation, J. Smith, P.M. Hunger, A.E. Donius, T.A. Freeman, and U. Wegst of Drexel University and the Thomas Jefferson University, USA. 
Addressing this gap, S.W. Lee (Stanford University), in symposium $P$, presented experimental results on the mechanical behavior of $\mathrm{Au}$ microcrystals prepared by solid-state dewetting of sputtered thin films, which are believed to contain low-to-zero initial dislocation densities. Lee's nanomechanical compression tests on these crystals revealed strengths near estimates of the ideal strength of $\mathrm{Au}$ in as-grown structures, significantly higher than similarly sized nanopillar counterparts prepared using focused ion beams. Lee reconciled this difference by show- ing that applying pre-strain to the microcrystals introduced dislocations which lowered the yield strength of the material, contrary to the common knowledge in bulk crystals that higher dislocation densities lead to higher strength.

Shape memory alloys (SMAs) are a unique class of materials with the ability to remember their original shape and withstand large, reversible deformation. While the mechanistic knowledge base on the phase transformations underpinning this response is mature for bulk materials, the effect of size on shape memory

\section{Graduate Students Receive Gold and Silver Awards}

Graduate Student Awards were announced during an evening ceremony on December 1, at the 2010 Materials Research Socety Fall Meeting in Boston.

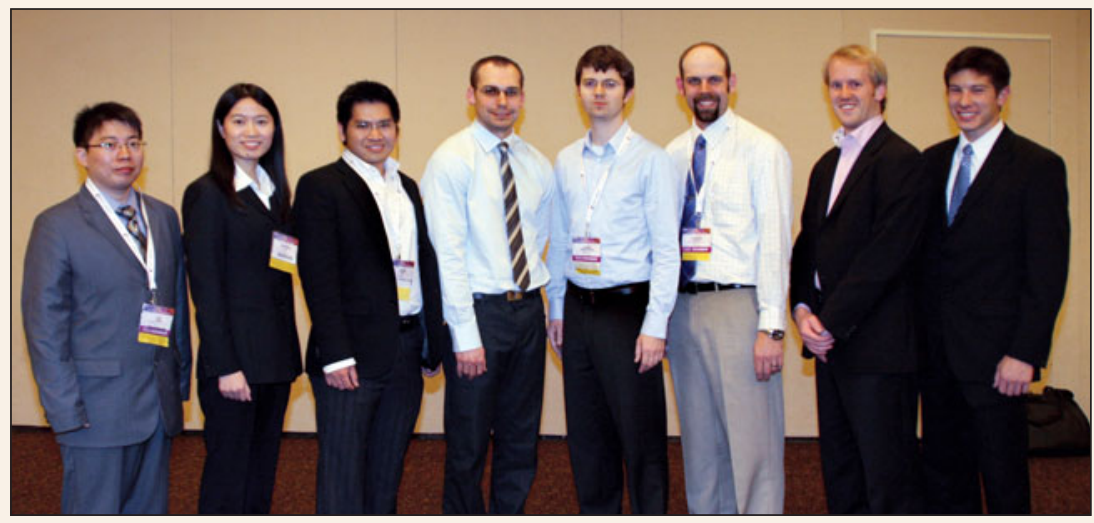

Gold Graduate Student Awards were awarded to (left to right): Lei Yang (Georgia Institute of Technology), Xiaoting Jia (Massachusetts Institute of Technology), Nhon Vo (University of Illinois, Urbana-Champaign), Andrew Bonifas (The Ohio State University), Lieven Verslegers (Stanford University), Stephen Morin (University of Wisconsin, Madison), Benjamin Almquist (Stanford University), and Daniel Shoemaker (University of California, Santa Barbara).

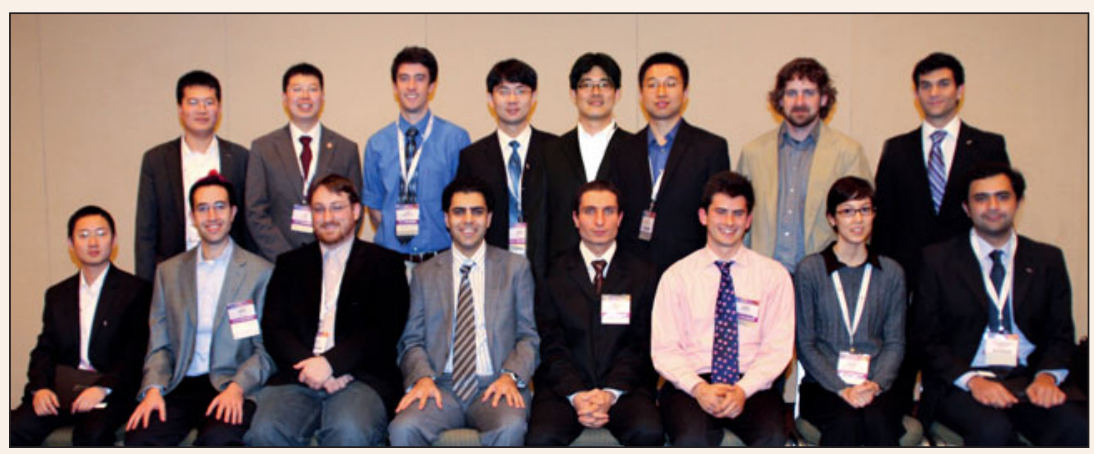

Silver Graduate Student Awards were awarded to (front row, left to right): Jingwei Bai (University of California, Los Angeles), Daniel Recht (Harvard University), Thomas Babinec (Harvard University), Rouzbeh Shahsavari (Massachusetts Institute of Technology), Michel Risser (INSA Strasbourg), James Neilson (University of California, Santa Barbara), Noemie Chocat (Massachusetts Institute of Technology), and Ravi Shankar Sundarum (Max Planck Institute for Solid State Research, Stuttgart); and (back row, left to right): Pu Yu (University of California, Berkeley), Lei Yang (Brown University), John Tumbleston (University of North Carolina, Chapel Hill), Guang Sheng (The Pennsylvania State University), Seung Hyub Baek (University of Wisconsin, Madison), Wenzhuo Wu (Georgia Institute of Technology), Edward Barry (Brandeis University), and Marco Righettoni (ETH Zurich). 
group III-V and group IV materials have many exciting applications prospects, including as solar cells and magnetic quantum dots-provided well-defined heterostructures can be achieved. In symposium W, F. Ross (IBM T.J. Watson Research Center) addressed the challenges of kinking and defects in the growth of nanowire heterostructure interfaces by studying in real-time the insertion of $\mathrm{Ge}$ into GaAs nanowires by in situ transmission electron microscopy (TEM). A twostage phase transformation was observed, where solid $\mathrm{Au}$ (used as a catalyst) first transforms into liquid $\mathrm{AuGe}$, and then Ge precipitates at the interface, reflecting changes in the catalyst as predicted by its phase diagram. From these observations, Ross provided a nucleation model for hybrid nanowire morphology to reflect both the layer-by-layer growth and the nucleation event at the nanowire edge that are possible upon incorporation of a second material. The nucleation event may lead to kinking, resulting in a change in the nanowire growth direction relative to the substrate. A survey of materials pairs suggested universal behavior to predict the growth mode that depends not on the relative lattice mismatch, but instead on the relative surface energy. Ross investigated side-branch morphology, explaining why some side-branches grow perpendicularly from the base nanowire while others crawl along the base nanowire. However, kinking may occur in the second material well after the heterostructure interface formation, which Ross speculated to be due to mismatch between the droplet volume and the area of the growth interface. The effect of diameter change on stacking fault formation was confirmed by adding more $\mathrm{Au}$ to a Si nanowire tip and observing the effect on nanowire growth morphology in situ.

Advances of nanotechnology will ultimately be enabled by the invention, development, and refinement of methods for the integration of nanomaterials into useful architectures and systems. Metamaterials enable control of resonant absorption and optical dispersion in metal-dielectric structures, resulting in new possibilities for solar energy conversion. In his talk in symposium
Y, H. Atwater (California Institute of Technology) discussed new structures and approaches with which new, functional systems have been created. One highlight was amorphous-silicon photovoltaics that were enhanced by
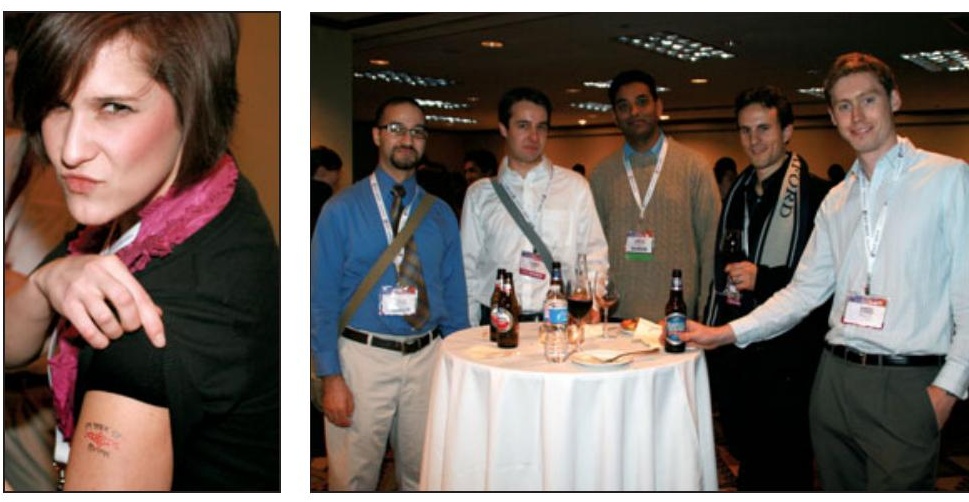
using plasmonic metamaterials as a patterned substrate. Blue light absorption was increased by the textured surface, while red light absorption was increased by resonance with the plasmonic metamaterial that coupled with light and reduced transmitted light. Since patterning was achieved using nano-imprint lithography, this solar energy system is lowcost, lightweight, and thin.

A. Javey (University of California, Berkeley) uses nanostructuring to enhance the efficiency of solar energyharvesting materials. Javey fabricated ultrathin III-V semiconductors by first growing 5-nm thick epitaxial InAs on a sacrificial layer and then transferring this to poly(dimethylsiloxane) (PDMS). The InAs was then transferred onto an oxidized silicon substrate. This allowed for tunable epitaxial III-V semiconductors on silicon that were much easier to fabricate, while performing in line with expectations. Javey then described the fabrication of large-area arrays of singlecrystalline nanopillars to create a photovoltaic device. By using a nanopillar structure, the surface area could be increased for collection and improved carrier transport. Varying diameters allowed for the reduction of surface and interfacial losses. Javey demonstrated how the photovoltaic device could be fabricated on flexible substrates. In a movie clip, Javey demonstrated how the output voltage of a flexible solar cell remained constant as the solar cell was flexed.

Advances in complementary metal oxide semiconductor (CMOS) devices are no longer restricted to Moore's law and the perpetual scaling to smaller sizes. The concept of "beyond Moore's law" describes the increasing functional diver- sification of CMOS. T. Mayer (the Pennsylvania State University) said that the future is moving beyond the confines of silicon processing to the incorporation of diverse nanomaterials that are processed in parallel. In her talk, Mayer discussed the integration of functionalized nanowires to combine sensing capabilities with CMOS architecture in a single, ultracompact, low-power platform. Nanowires can be used as sensors to produce either mechanical or electrical signals that change in response to the binding of a target molecule. To be compatible with CMOS, these nanowires need to be deterministically placed on a prefabricated device in order to be contacted and integrated. Mayer accomplished this through patterning wires on the substrate and creating electric fields that directed the assembly of the nanowires to specific regions of the chip. Repulsive electrostatic forces of the nanowires ensured uniform spacing. Mayer showed how various kinds of nanowires could be placed accurately by introducing them in series, with yields exceeding $90 \%$ with a less than $1 \%$ mismatch across three populations of DNA-coated nanowires.

The aperiodic, disordered structures of aerogels and aerogel-inspired materials show immense promise as functional porous materials. Carbon aerogels have high surface areas and tunable porosities, while being electrically conductive, monolithic, and chemically robust. These materials are being investigated for a variety of applications, including catalysis, adsorption, and energy storage. In symposium BB, T. Baumann (Lawrence Livermore National Laboratory) described his exploration of modifying and demonstrated an integrated device 

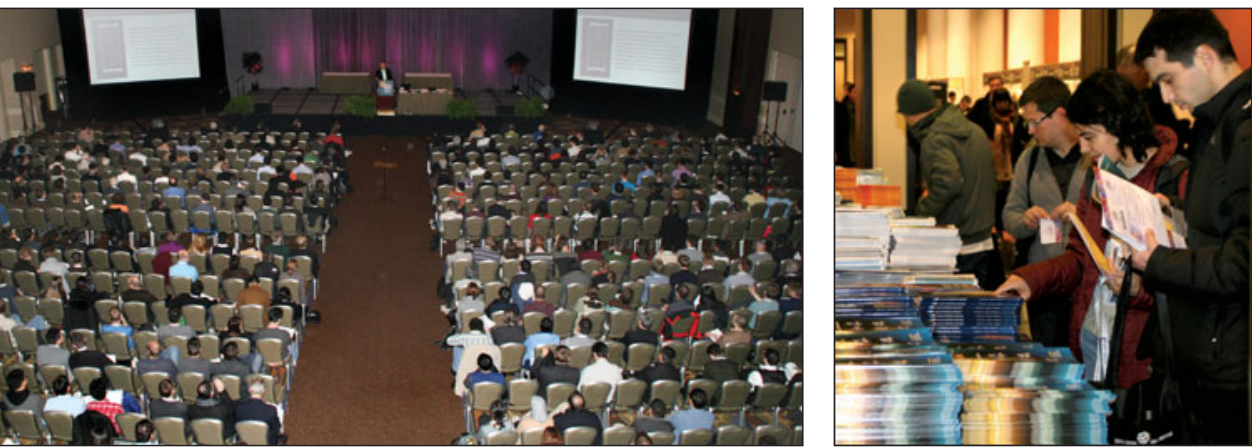

the carbon aerogel process by adding precursors and performing post-synthetic modifications through additives. First, Baumann discussed carbon nanotubeand graphene-based aerogels, using a sol-gel as an electrically conductive nanoglue to stabilize the carbon structures. The results were improvements in thermal and electrical conductivities while being stiff and low density at small strains and superelastic at large strains. Baumann used a controlled burn-off of carbon to create ultrahigh surface areas, making the structure ideal for hydrogen adsorption. He demonstrated a $5 \mathrm{wt} \%$ hydrogen adsorption, which is comparable to the best carbon-based adsorbates. Baumann illustrated other techniques to engineer carbon aerogel properties, such as atomic layer deposition of Pt to allow for catalysis which showed $100 \%$ efficiency in oxidizing $\mathrm{CO}$ to $\mathrm{CO}_{2}$, and carbon nanotube growth through chemical vapor deposition to increase electrical conductivity.

Crystallographic alignment in thin films or nanostructures on top of polycrystalline or amorphous substrates is beneficial for improved properties of functional materials used in various applications. Numerous techniques are being applied to achieve long-range order texturing such as, for example, ion-beamassisted deposition (IBAD), oblique angle deposition, ion beam treatment, and surface templating. During symposium DD on artificially induced crystalline alignment in thin films and nanostructures, R. Spolenak and co-workers (ETH Zurich, Switzerland) presented two successful strategies to induce a texture in thin metal layers, namely, high energy $(\mathrm{MeV})$ post-deposition ion irradiation and selective sputtering of thin films at low energy (keV). V. Matias (Los Alamos National Laboratory) showed the latest results of the IBAD technique, where in-plane alignment of $1.6^{\circ}$ full width at half maximum was achieved in $\mathrm{MgO}$ thin films grown on metal tapes. Combinatorial experiments helped shed more light on the texturing process itself. The IBAD technique has been successfully used for the preparation of textured templates for high-temperature superconductors, but also for other materials. In particular, B.M. Clemens (Stanford University) utilized the IBAD technique to prepare textured $\mathrm{CaF}_{2}$ template layers to realize highly efficient solar cells. Texture development during inclined sputtering was explored by the group of D. Depla (Ghent University, Belgium). The researchers achieved biaxial alignment in several materials ( $\mathrm{TiN}, \mathrm{MgO}$, YSZ, InN, and Cr) under specific conditions. They also introduced an analytical model supported by Monte-Carlo simulations, which allowed for an understanding of the texturing mechanism.

\section{Energy, safety, and materials exploration}

Mass marketing of fuel cells is often limited by high component costs and inadequate durability in service. In symposium GG, E. Wachsman (University of Maryland) said that while proton-exchange membrane fuel cells will ride the ups and downs of perceptions in the hydrogen economy, solidoxide fuel cells (SOFCs) have a more stable prospect as an energy conversion device since SOFCs run on other fuels. One main research area in SOFCs is in reducing the operating temperature from $800^{\circ} \mathrm{C}$ to less than $600^{\circ} \mathrm{C}$, which would reduce cost and increase reliability. In or- der to reduce the operating temperature, Wachsman focused on higher conductivity and lower temperature bismuth oxide electrolytes. A bilayer of bismuth-oxide/ ceria electrolyte was found to overcome reactions with a reducing environment, while increasing power density by over $35 \%$. A bismuth-ruthenate/bismuth-oxide composite cathode could increase both electronic and ionic conductivities. U1timately, Wachsman was able to demonstrate a fuel cell with both components that attained power densities of $\sim 2 \mathrm{~W}$ / $\mathrm{cm}^{2}$ at $650^{\circ} \mathrm{C}$, which has sufficient power down to $400^{\circ} \mathrm{C}$ and potentially opens SOFCs for transportation and portable power applications.

Photovoltaic systems based on organic materials and nanoparticle hybrid technologies are currently attracting widespread attention because of their potential for low-cost and high-throughput manufacturing. In symposium JJ on nanostructured polymeric materials, M. Durstock (U.S. Air Force Research Laboratory) discussed his work on understanding and designing the surfaces of mesoporous titania films for dye molecules. After discussing layer-by-layer dye assembly, Durstock delved into the creation of a large-scale array of vertically aligned titania nanotubes of controllable shape and size. The array was created by first anodizing alumina into nanoporous structures, filling the pores with $\mathrm{TiO}_{2}$ and then removing the $\mathrm{Al}_{2} \mathrm{O}_{3}$ template. Using a roughened surface allowed him to increase the dye concentration on the surface. Durstock was able to demonstrate power conversion efficiencies of $5.4 \%$, revealing good efficiency given the smaller surface area as compared to commercial devices.

Somewhere between increasing energy demand, rising $\mathrm{CO}_{2}$ emissions, and increased global warming, M. Dresselhaus (Massachusetts Institute of Technology) believes that thermoelectrics will play a major role. In symposium LL, she outlined some major trends that she has been observing over the years in thermoelectrics that will continue to shape the technology, such as engineering at the nanoscale to independently control parameters, as well as including particles 
to give additional scattering and capture additional frequencies. Dresselhaus urged researchers to keep pushing the envelope in trying new materials and testing new concepts, reassuring the audience that funding agencies are okay with "crazy ideas." J. Fairbanks of the U.S. Department of Energy described specific topics of nine new programs in thermoelectric materials and device research that were recently funded by a National Science Foundation-Department of Energy partnership in thermoelectrics.

The new scientific frontier of nanotechnology comes along with the realization that nanoscale materials can easily find paths to enter biological organisms and penetrate to the subcellular levels. In symposium RR, K.A. Dawson (University College Dublin, Ireland) overviewed aspects of safety and nanotoxicity of nanoscale materials that are becoming critical issues. Dawson said that there has been an incoherent approach to safety in nanotechnology. The first reports appeared in 2002. The first few years represented a state of confusion. Currently, we are in a state of stabilization, he said. It is important to recognize that in a biological system, while chemicals are partitioned, nanoparticles are processed. Nanoparticles can travel into cells through existing pathways. However, nanoparticles can be completely tracked within a biological system - they cannot be lost. Dawson presented several examples of related research, concluding that scientific study will be able to guide society to "nanosafety," which is crucial for the success of nanotechnology.

Polymer-based smart materials are being incorporated in a variety of applications ranging from artificial muscles to reusable Braille dots on surfaces to camera lenses for smart phones to applications in harvesting energy from wind, waves, and human motion. In his talk in symposium HH, I. Anderson, from the Biomimetics Laboratory at the University of Aukland in New Zealand, presented a device that has the potential to produce dielectric elastomer generators from low-cost acrylic membranes and carbon grease mounted in a frame. This type of device could be used to power mobile devices using wearable energy harvesters that could harvest energy from the human gait. Applications for reusable Braille dots using hydrostatically coupled dielectric elastomer actuators (HC-DEA) were presented by F. Carpi of the University of Pisa, Italy. HC-DEAs are based on an incompressible fluid that mechanically couples a DE-based active part to a passive part interfaced to the load, so as to enable hydrostatic transmission which can be electrically controlled. An 8-dot refreshable cell for dynamic Braille displays and a wearable tactile display used to provide users with tactile feedback during electronic navigation in virtual environments are two applications that show the potential of the new technology and the prospective opportunities for haptic interfaces.

In symposium SS on advanced imaging and scattering techniques for in situ studies, J. Kirz (Lawrence Berkeley National Laboratory) said that x-rays are an important tool to visualize the nanoworld, because of their short wavelengths, good penetration, and contrast through absorption edges. Zone plates are an important part of x-ray microscopes for diffractive optics. Currently, microscopes are "sprouting" at all synchrotron radiation sources. The current trend is moving from two- to three-dimensional studies such as in spectromicroscopy, tomography, and time-resolved analysis. For example, using a new double patterned gold zone plate, $12 \mathrm{~nm}$ resolution has been achieved. Kirz contemplated a new approach by getting rid of optics altogether, by using a computer to phase the scattered light rather than a lens with some prior knowledge of the object and an appropriate algorithm. He described one example of imaging a freeze-dried yeast cell, where a threedimensional image could be constructed from diffraction data by oversampling. Several other lensless methods have also been demonstrated. Kirz concluded by suggesting that lensless methods of exploration is a rapidly evolving field with much potential.

The symposium also featured a panel discussion with eight experts from academia, industry, national laboratories, and funding agencies presenting on future directions for scanning probe microscopy. Themes included opportunities for higher speed data acquisition and imaging, measurements based on functional tips, systems combining SPM and other techniques for simultaneous experiments, and further expansion to chemical and biological studies. "Big picture" goals of chemically identifying unknown nanovolumes or even atoms with SPM were also widely agreed upon, along with the value of long-lasting, sharp (or even selfsharpening) probes. Other topics emphasized by the panel and the audience included the importance of nano-related standards, the benefits of establishing procedures for common measurements, and the need for sustained funding opportunities. There was overall acknowledgment that the term "quantitative" is [mis] used far too widely; and that SPM must be the second most important materials science advance in the last 50 years. Ultimately, as John Pethica (Trinity College, Dublin) reminded the audience near the end of the session, the progress in this still young discipline ( $<30$ years) has been remarkable, and the promise of future developments and applications are exciting.

\section{Energy policy and materials research}

Energy is clearly one of the critical global challenges facing humankind. And solutions involve science, including materi-
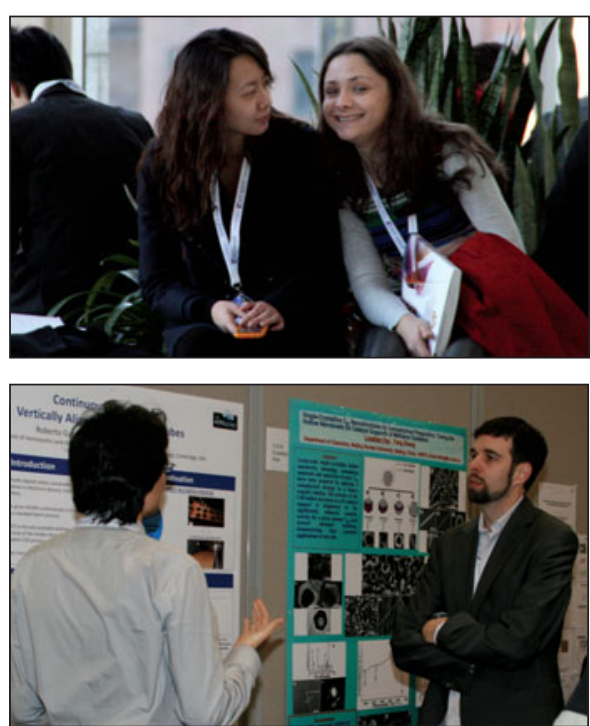


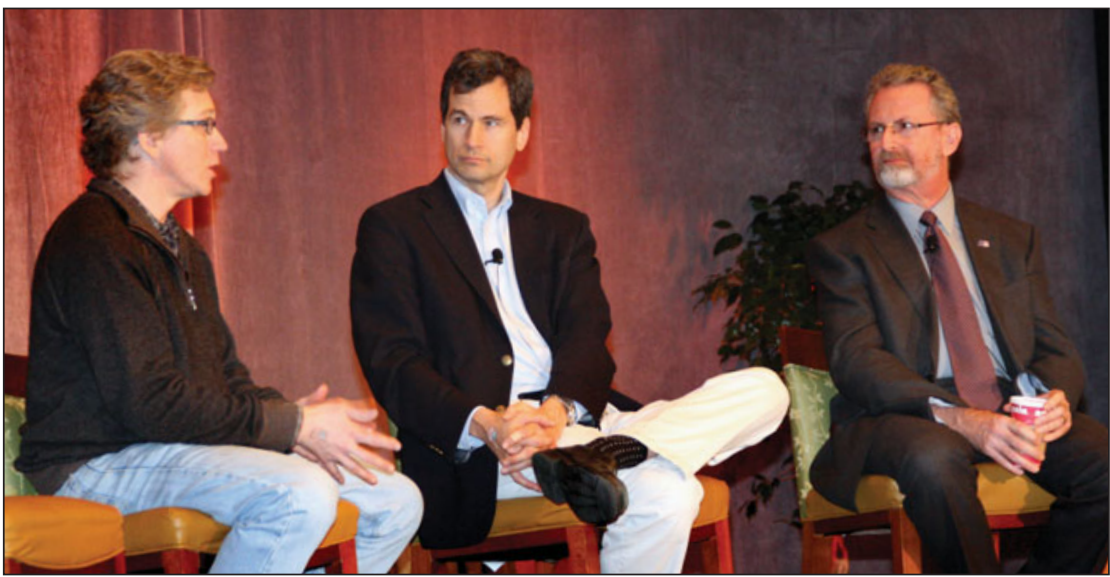

als research, as well as economic and political implications. During the MRS Meeting, Eberhard Umbach, president of the Karlsruhe Institute of Technology in Germany, discussed the current energy landscape in Europe; a science policy forum addressed a study on energy critical elements; and Christopher P.J. Barty, Chief Technology Officer of the National Ignition Facility at Lawrence Livermore National Laboratory (LLNL), discussed the path to laser inertial fusion.

There is a significant diversity in energy usage among the countries in the European Union. However, this should not inhibit the development of a common European strategy in energy politics, especially energy research politics, said Umbach. Umbach noted the connection between electricity consumption and gross national product (GNP) per capita. The higher the GNP, the higher is the electricity consumption. Norway has the highest electricity consumption and almost the highest GNP per capita. Norway also has the highest hydroelectric power generation, Umbach said. Renewables have been successfully incorporated into the European energy picture, and their use is anticipated to grow, particularly wind energy.

Umbach discussed several fields of research including efficient energy conversion and use, carbon capture/ storage, carbon dioxide reuse, electrochemical storage, concentrating solar power, smart grids, next-generation biomass, geothermal energy, photovoltaics, nuclear power, and fusion. He said that materials issues are pervasive through all of these areas, and materials research is crucial in each. In the area of efficient energy use, for example, he described a new cement, celitement, as a replacement for conventional Portland cement, made of an hydraulic adhesive agent based on calcium silicates. The use of celitement could reduce associated energy consumption by $50 \%$ and also reduce $\mathrm{CO}_{2}$ emission by $50 \%$. Umbach advocates energy research for a sustainable energy supply with an optimal energy mix. All technological options must be considered, he said.

Members of a committee studying energy critical elements (ECE), convened in 2010 by the American Physical Society and the Materials Research Society, conducted a panel discussion in the venue of a Science Policy Forum. The purpose of the joint policy study is to evaluate constraints on availability of energy critical elements that might inhibit the large scale deployment of new technologies for the production, transmission, efficient use, or conservation of energy, and to make recommendations that would help avoid those obstructions. release of the report, speakers carefully avoided findings in the ECE report but rather covered individual perspectives informed by their work on the committee. Co-chair Robert Jaffe, Morningstar Professor of Physics at the Massachusetts Institute of Technology, was joined by committee members Alex King, director of Ames Laboratory; Alan Hurd, director of the Lujan Center at Los Alamos; and Ron Kelley, MRS Washington consultant who also served as forum moderator.

Widely reported stories about China's
As the Forum was held prior to the near-complete control of the rare earth (RE) market have gripped everyone's attention for over a year. Almost weekly workshops dealing with the RE crisis have become even more frequent since China's announcement in July 2010 of dramatically lowered export quotas. The price volatility of RE oxides, inflating from double digits to triple digits within months, was cited by panelists as especially vexing to long-term business planning. King said, "Thermodynamics counts, but economics controls."

Jaffe said an "energy-critical" elements category is identified as having "the potential for major impact on energy systems and for which significantly increased demand may come as a novelty and perhaps a shock." This definition is at once broader than rare earths and surgically narrower than traditional energy materials such as nuclear materials. Jaffe cited tellurium, much rarer than rare earths, as a case study. Essential to thinfilm CdTe solar cells, known Te reserves limit the technology to only $6 \%$ of current electricity demand, all other things being constant. However, economics and geopolitics could blunt the utilization of CdTe cells significantly given the availability of polluting alternatives. Similar considerations jeopardize dozens of new energy technologies.

Another case study is neodymium. Neodymium-based high strength magnets enable efficient wind energy, hybrid cars, and many defense-related technologies. Although national security considerations were outside the scope of the study, Hurd emphasized the complex connections of ECEs in dual-use demand. Materials researchers seeking replacements for ECEs may be able to extend critical resources: Recently reported mixed rare-earth magnets use less Nd while improving on temperature range.

King echoed testimony to the committee that training a new generation of experts, from geoscientists to energymaterials researchers, is essential. A large fraction of the Western World's expertise in rare earths resides at Ames Laboratory, but in retirement. Although the current crisis will probably be temporary, its effects could be long lasting until education 
catches up with demand, said King.

In discussion, the unique issues surrounding helium arose. The ECE committee took up He as a special case involving stockpiling. While stockpiling can disrupt markets in unpredictable, undesirable ways, He is an exception for good reasons. The United States accumulated a large He stockpile during much of the 20th century only to begin to sell it off in 1995 to pay for the price of inventory. Helium's unique physical properties render it irreplaceable in energy research and applications, such as advanced nuclear reactors.

The APS-MRS report was issued jointly by the APS Panel on Public Affairs and the MRS Government Affairs Committee in February 2011. It can now be downloaded online from www.mrs. org/advocacy/ece.

The National Ignition Facility (NIF) is a major project of the U.S. Department of Energy (DOE) to demonstrate laser fusion and pave the way to laser inertial fusion as a viable power source. The NIF is now operational and is the largest scientific construction project completed by DOE, said plenary speaker Christopher P.J. Barty, Chief Technology Officer of the NIF. The facility, located at the Lawrence Livermore National Laboratory (LLNL) has 192 laser beams, and it is, overall, 50 times more energetic than any other laser, he said. This laser energy is focused into a spot with a $\mathrm{mm}^{3}$ volume. It is currently operating at $1.3 \mathrm{MJ}$ energy while the target is $1.8 \mathrm{MJ}$. The NIF missions include national security, advancing frontier science, education, and enabling clean energy.

Barty said that once NIF achieves its ultimate goal, the next step is laser inertial fusion energy (LIFE), the planning for which has already started. NIF will allow the physics risks to be retired for LIFE which could be the ultimate energy source, he said. A number of challenges for LIFE are materials-related, including the first wall material, fission fuel, laser, hohlraum, and target shell, so materials researchers have an important role to play in this endeavor.

NIF is designed to produce fusion burn and energy gain using a technique known as inertial confinement fusion. NIF's intense laser beams, focused into the hohlraum - a centimeter-scale, can-shaped container made with highatomic-mass materials like gold and lead with holes in the ends for beam entrywill generate a "bath" of soft x-rays that will compress a tiny hollow shell filled with deuterium and tritium to 100 times the density of lead. In the resulting conditions - a temperature of more than 100 million degrees Celsius and pressures 100 billion times the Earth's atmosphere - the fuel core will ignite and thermonuclear burn will quickly spread through the compressed fuel, releasing 10 to 100 times more energy than the amount deposited by the laser beams.

Barty said that NIF could be the path to eventual clean, economically viable fusion energy to replace all current fossilfuel based and other sources.

\section{Special talks and events}

In addition to the technical program, the Meeting presented the plenary and award talks, technical talks for a general materials audience, presentations on outreach, and on U.S. funding opportunities. L. Eric Cross of the Pennsylvania State University, who received the highest honor of the Materials Research Society, the Von Hippel Award, discussed the use of flexoelectric composites as a viable option for a new class of lead-free piezoceramics. Piezoelectric properties get stronger with decreasing scale of the texture symmetry. Importantly, direct and converse piezoelectric properties can be separately controlled to yield sensors that will not actuate and actuators that will not sense. This is useful as properties of current smart materials. Also, he said, current indications are that the best flexoelectric piezoelectric composites will be lead-free. Cross described a bio- logical biomembrane system that exhibits effective flexoelectricity. This is a liquid crystal-like membrane in the human ear that exhibits both a direct and converse effect, and it turns out that this is crucial for human hearing.

David D. Awschalom of the University of California, Santa Barbara delivered the David Turnbull Lecture in which he described the strong interplay between materials science and physics that has driven scientific progress in semiconductor spintronics and quantum information processing from low-temperature measurements on spin ensembles to room temperature studies of single spins. Among examples of unexpected discoveries, Awschalom described a surprise measurement that had an enormous impact in the field of spintronics, a simple nonmagnetic control experiment in GaAs revealed that coherent electron states could be generated and maintained for many orders of magnitude longer in time than predicted ( $\sim 150 \mathrm{~ns})$. These experiments opened the door to new methods for manipulating spin including electrical control through the spin-orbit interaction, observation of the spin Hall effect, and the identification of new pathways to control nuclear spins in the solid state. These latter mechanisms included the discovery of ferromagnetic imprinting, where nanometer-scale thick ferromagnetic films are used to imprint nuclear polarization in the solid state through spin-polarized
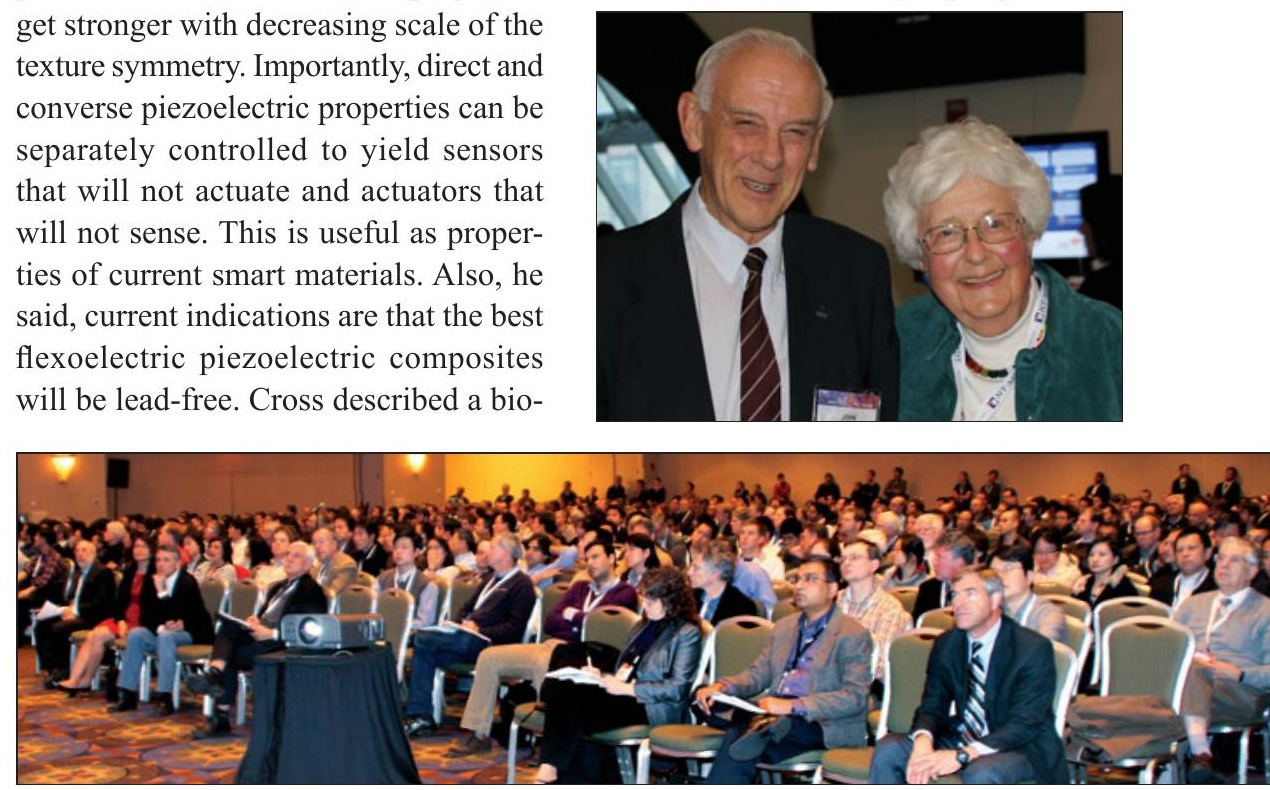
electrons. Among recent developments, Awschalom described how impurity states in diamond, another semiconductor, are used to trap individual electron states at room temperature. He discussed experiments that use on-chip circuits to manipulate these single electron spins at gigahertz frequencies, and exploit ion implantation recipes to fabricate arrays of spins for possible applications in quantum information processing. Awschalom concluded with an outlook toward the future and prospects for "quantum computing with defects."

Charles M. Lieber of Harvard University, who received the Kavli Distinguished Lecture in Nanoscience, showed that nanowires were an ideal platform for exploring scientific possibilities and building new technologies. Key advances in controlled nanowire synthesis were used to build function, he said. Controlling the radial growth and composition of nanowires led to Ge/Si core-shell structures that enable transistors that exceed the limits of top-down devices, have currents that approach the ballistic limit, and provide nanostructures for quantum computing, among other applications. Along with the core-shell nanowires, controlling the growth and composition in the axial direction provide nanostructured materials that can be used in a gen-

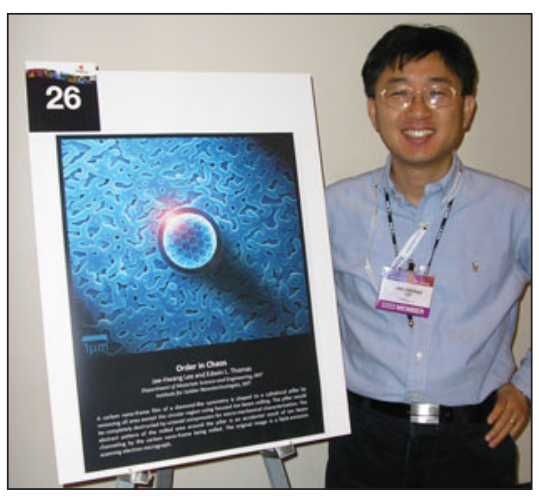

eral approach to improve photovoltaics, enable fundamental studies at the level of a single nanodevice, and eventually lead to integrated photovoltaic power elements in self-powered nanoelectronics. Lieber also examined nanoelectronicbiology interfaces.

MRS Medalist Walter A. de Heer of Georgia Institute of Technology presented a lecture on graphene, reported at the beginning of this article. The Woody Award, presented annually by the MRS President to recognize an outstanding MRS volunteer, as exemplified by Woody White, was given to Michael J. Aziz of Harvard University.

In Symposium X, the lunchtime series of talks for the nonspecialist, John A. Rogers of the University of Illinois at Urbana/Champaign addressed biointegrated electronics. He summarized fundamental and applied research on semiconductor technologies that can integrate intimately with biological tissues (bio-integrated electronics) or that can resorb at programmable rates in them (bio-resorbable electronics). Major technical and scientific challenges are in the realization of bio-compatible interfaces between the classes of devices that might be most useful in these contexts and the soft, curvilinear surfaces of the body. These ideas can be exploited in two classes of application: minimally invasive surgical tools and diagnostic systems that conformally wrap internal organs, to provide advanced functionality in cardiology and neurology; and monitoring and therapeutic electronics that can be placed into the body and then disappear, in part or entirely, as they are metabolized.

Jennifer Stancil, Executive Director of Educational Partnerships at WQED Multimedia, spoke at the Women in Materi-

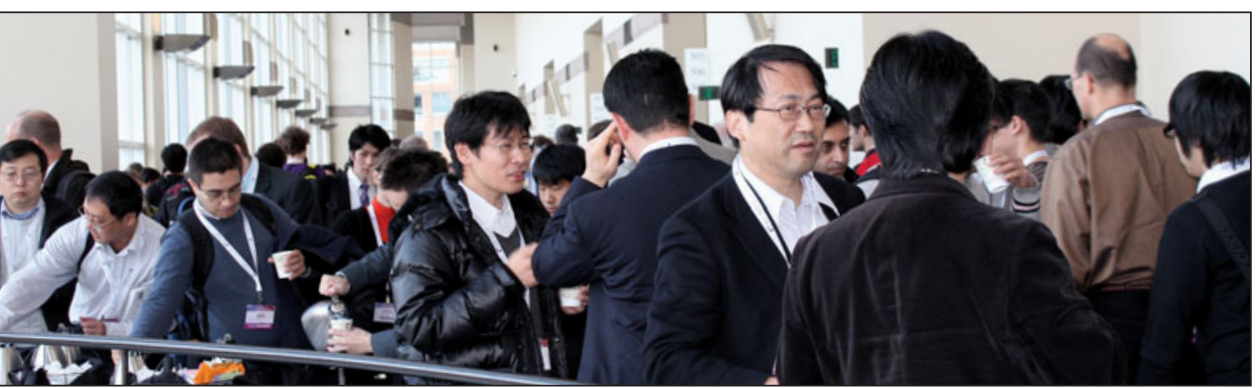

als Science and Engineering Breakfast. In her presentation, "Girls in STEMInspiring the Next Generation," Stancil focused on four key areas: The Science Slump, What We Know about Girls, A Comparison Model for Materials Science to engage girls, and a Call to Action. Key to engaging girls in science, she said, is to tailor the curriculum to the way girls think, learn, and grow. For example, children ages $10-18$ are very tied to social media. However, girls use social media to communicate with others and prefer audio while boys tend to use social media for video games. Girls think and learn through group work. Therefore, science venues such as science fairs involving group activities can be co-opted to engage girls. Also, scientists are typically presented to the public as individuals; girls may be more attracted to the field if they see visuals of groups of scientists collaborating in the laboratory. Girls' attraction to the field of materials research may additionally be enhanced if they saw their participation as part of a larger goal, such as ways to avert natural disasters. During a question/answer session, Stancil further suggested some points where the Materials Research Society can differentiate itself as it grows its outreach to secure a diverse and passionate workforce for the 21 st century.

During the course of the week, speakers from several U.S. government agencies presented opportunities to obtain funding for materials research. The opportunities range from curiosity-driven basic research within the Division of Materials Research at the National Science Foundation, to curiosity-driven research in energy through the Materials Sciences and Engineering Division within the Office of Basic Energy Sciences of the Department of Energy and in biomaterials through the National Institute of Biomedical Imaging and Bioengineering (NIBIB) at the National Institutes of Health. Other opportunities are more focused such as high-risk, high-reward fundamental research specifically for defense needs funded through the Defense Advanced Research Projects Agency (DARPA) and high-risk, high-reward translational research that is expected to 
transform the United States' capacity to address areas of critical national needs as funded by the Technology Innovation Program (TIP) at the National Institute of Standards and Technology.

\section{MRS committee news}

Materials activities and opportunities in Africa

There is increasing interest in materials science and materials research in Africa. Africa-MRS was formed about a decade ago and is being actively developed. A special round-table discussion was held at the MRS Fall Meeting for attendees to learn about opportunities in international collaborative materials-related activities in Africa. MRS has an Africa subcommittee with Eric Garfunkel as chair, under the umbrella of the MRS International Relations Committee (IRC). Garfunkel opened the session describing the charges for his subcommittee. Julia Hsu, chair of the IRC, presented some brief comments. Next, Josephat Zimba, President of Africa MRS, overviewed the origins and launch of Africa-MRS. Africa MRS is 10 years old, and is beginning to meet with other groups to plan meetings, workshops, and summer schools, as well as develop education, research, and outreach projects. Zimba invited everyone in the audience to attend the next Africa MRS conference in December, 2011, in Victoria Falls, Zimbabwe.

Both MRS Executive Director Todd Osman and MRS President David Ginley described the motivations for MRS to be involved with materials-related activities in Africa. They emphasized that this is a crucial activity that will positively impact materials research. Zakya Kafafi from the National Science Foundation (NSF) discussed some of the activities supported by NSF relating to materials research in Africa. NSF is fully supportive of the efforts of MRS and Africa-MRS. She underscored the importance of understanding other cultures before getting involved with them in collaborative activities. Abdelilah Slaoui, representing the European-MRS, gave the European perspective. Sossina Haile (California Institute of Technology) described successful efforts in setting up a Materials
School in Ethiopia with NSF support to allow U.S. materials researchers to visit and teach.

The presentations were followed by an open discussion. A student from Kenya stated that the biggest obstacle for African students is motivation. HannsUlrich Habermeier (Max-Planck-Inst.) suggested that the Africans list their needs since they understand them best. The importance of materials science and engineering students being able to find local jobs was raised. There is also concern in Africa that there is fear of losing their brightest students to the West. It was pointed out that there is significant investment into Africa and jobs are sure to follow; investment is doubling every five years. The importance of involving industry in discussions and conferences was recommended. A suggestion was made to send the MRS Strange Matter exhibit to African countries in particular to educate younger Africans about materials science and encourage them to pursue the field. Professional societies can play a major role in helping scientists and researchers in Africa network with colleagues within Africa as well as outside. Kafafi suggested including the Middle East in discussions about materials science in Africa, since there is a large thrust in funding and setting up of institutions there.

\section{NOVA Series-MAKING STUFF}

Can you imagine a world where tiny robots can be injected into an eye, moved precisely to clotted blood vessels and deliver a small dose of drugs to heal blindness? What about a world where chicken feathers help store hydrogen for the next generation of cars? It turns out that such imagination would not have to wander much outside of the realm of MRS and materials science. And that furthermore, David Pogue, the television host for the show MAKING STUFF, finds that these amazing stories are fun, entertaining, and maybe even a little educational. $M A K-$ ING STUFF is a four-part television series on NOVA, a science program on public television, that takes viewers on a journey through the materials science world where things get stronger, smaller,
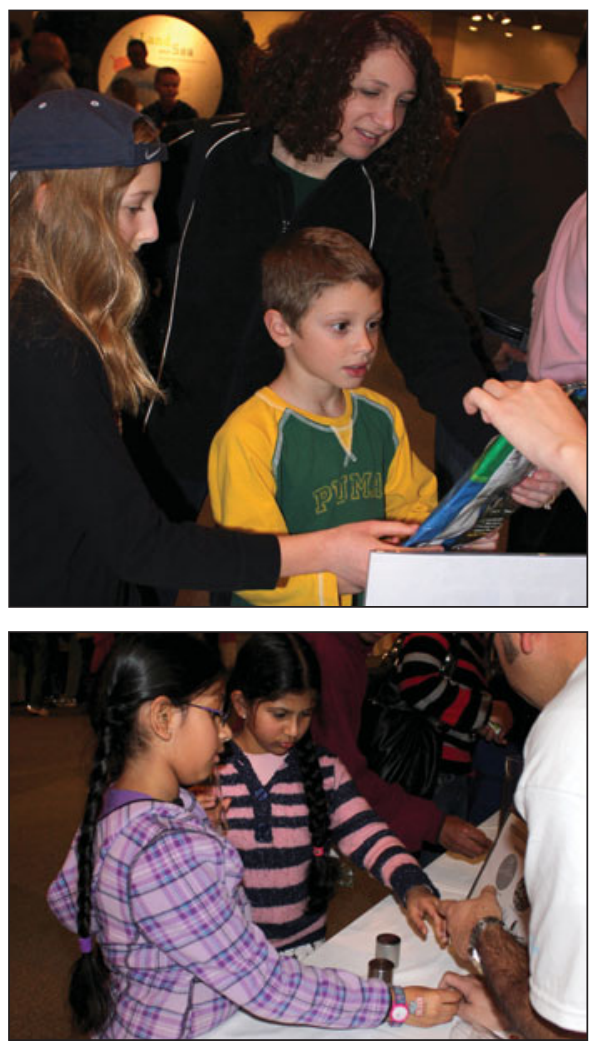

cleaner, and smarter. The series began airing on January 19, 2011 in the United States, with plans to also be shown internationally. During the MRS Meeting, the MRS Outreach Committee hosted a panel discussion on the series.

Joining Pogue in the panel discussion were NOVA producer, Chris Schmitt; Alex King, director of the Ames Laboratory; and Amy Moll, Boise State University, who is chair of the MRS Outreach Committee. The group outlined the challenges of articulating materials science through a television show that does not contain any words beyond what eighthgraders would understand, and they mused about their favorite segments. The audience was treated to a preview of the show for a taste of what to expect in January when the show actually aired. Lively question and answer sessions were conducted between the previews.

MAKING STUFF is produced in cooperation with the Materials Research Society. Major funding for MAKING $S T U F F$ is provided by the National Science Foundation, and additional funding is provided by the Department of Energy. Information on the series can be accessed at www.pbs.org/wgbh/nova. 